\title{
ОСОБЕННОСТИ ВЫЗВАННОЙ АКТИВНОСТИ МОЗГА ДЕВУШЕК-НОСИТЕЛЬНИЦ РАЗЛИЧНЫХ ГЕНОТИПОВ ПО МАРКЕРУ МАОА - UVNTR ПРИ ОЦЕНКЕ ЭМОЦИОНАЛЬНО ОКРАШЕННЫХ СЦЕН
}

\author{
Павел Н. Ермаков, Екатерина М. Ковш", Елена В. Воробьева
}

Южный федеральный университет, г. Ростов-на-Дону, Российская Федерация *E-mail:emkovsh@sfedu.ru

Работа выполнена при финансовой поддержке Российского научного фонда (проект № 16-18-10222 «Агрессивные и враждебные поведенческие стратегии у лиц с разными ДНК-маркерами»)

Изучение связей между особенностями строения генов и параметрами вызванной активности мозга - актуальная тема современных исследований. Известно, что ген МАОА, оказывающий влияние на концентрацию таких нейромедиаторов-моноаминов, как дофамин, серотонин и норадреналин, ассоциирован с различными психологическими характеристиками. Однако исследовательских работ, посвященных изучению ассоциации данного гена с психофизиологическими показателями, на сегодняшний день недостаточно. Целью данного исследования явилось изучение особенностей амплитудных и пространственно-временных характеристик вызванной активности мозга девушек 18-24 лет, являющихся обладательницами различных генотипов МАОА. По результатам генотипирования участницы (61 человек) были разделены на две группы: с высокоактивными и гетерозиготными генотипами по маркеру MAOA -uVNTR. В ходе исследования была проведена регистрация зрительных вызванных потенциалов в 64 оmведениях. В качестве стимулов выступили эмочионально окрашенные и нейтральные изображения.

В работе описаны современные представления о связи генотипов МАОА с особенностями кортико-лимбического взаимодействия. По результатам собственного исследования получено, что гетерозиготные генотипы, вероятно, связаны с меньшей эффективностью регуляторных функций. Для активации нейронных систем лобных отделов девушкам-носительницам гетерозиготных генотипов МАОА необходимо воздействие раздражителей, обладающих высоким arousal-эффектом, что может быть связано с менее эффективным прочессом отбора релевантных стимулов. В то же время, высокоактивные генотипы МАОА у женщин, вероятно, ассоциированы 


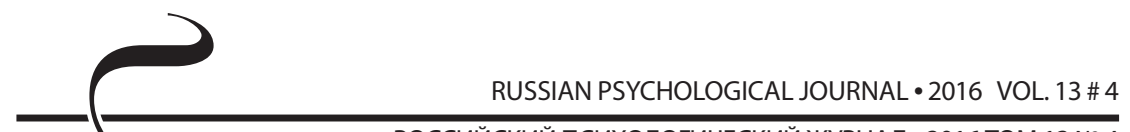

РОССИЙСКИЙ ПСИХОЛОГИЧЕСКИЙ ЖУРНАЛ • 2016 ТОМ 13 № 4

с высокой эффективностью управляющих функций (внимание, планирование, контроль), выполняемых префронтальными отделами коры мозга при оценке эмоционально окрашенных изображений, что сопровождается достаточной интенсивностью активации данных мозговых структур.

Ключевые слова: вызванные потенциалы, психогенетика, ген МАОА, моноаминоксидаза А, генетический маркер, зрительные стимулы, эмоции, управляющие функции, префронтальные отделы, нейромедиаторы.

Для цитирования: Ермаков П. Н., Ковш Е. М., Воробьева Е. В. Особенности вызванной активности мозга девушек-носительниц различных генотипов по маркеру MAOA -uVNTR при оценке эмоционально окрашенных сцен // Российский психологический журнал. - 2016. - Т. 13. - № 4. - С. 232-253.

Материалы статьи получены 27.10.2016

UDC 159.91

doi: 10.21702/rpj.2016.4.14

\section{FEATURES OF THE EVOKED BRAIN ACTIVITY IN FEMALE CARRIERS OF DIFFERENT MAOA -UVNTR GENOTYPES WHEN ASSESSING EMOTIONALLY CHARGED SCENES}

\section{Pavel N. Ermakov, Ekaterina M. Kovsh", Elena V. Vorobyeva}

Southern Federal University, Rostov-on-Don, Russian Federation

* Correspondence author. E-mail: emkovsh@sfedu.ru

\section{Acknowledgments}

The study was supported by the Russian Science Foundation (grant 16-18-10222 "Aggressive and Hostile Behavioral Strategies in Individuals with Different DNA Markers")

Studying the correlation between the characteristics of the structure of genes and the parameters of the evoked brain activity is a relevant problem of modern research. It is known that the MAOA gene, which influences the concentration of such neurotransmitters-monoamines as dopamine, serotonin, and norepinephrine, is associated with different psychological characteristics. However, very little is known about the association of this gene with psycho-physiological indices. The purpose of the present study was to investigate the characteristics of amplitude and spatial-temporal characteristics of the evoked brain activity in girls (at the age from 18 to 24) who were carriers of different MAOA genotypes. According to the results of genotyping, the participants (61 persons) were divided into two groups: with the 
high-activity and heterozygous MAOA -uVNTR genotypes. In the study the authors recorded visual evoked potentials in 64 derivations. Emotionally charged and neutral images were stimuli in the study.

The paper describes modern views on the correlation between MAOA genotypes and the features of cortico-limbic interaction. The results of the study showed that the heterozygous genotypes were apparently associated with lesser efficiency of regulating functions. To activate neural systems of the frontal divisions the female carriers of the heterozygous genotypes of MAOA needed the stimuli with a high arousal effect; this can be connected with a less efficient process of selecting relevant stimuli. At the same time, the high-activity MAOA genotypes in women are probably associated with high efficiency of controlling functions (attention, planning, control) of the prefrontal cortex when assessing emotionally charged images, which is accompanied by the intensity of activation of these brain structures.

Keywords: evoked potentials, psychogenetics, MAOA gene, monoamine oxidase A, genetic marker, visual stimuli, emotions, controlling functions, prefrontal divisions, neurotransmitters.

For citation: Ermakov P. N., Kovsh E. M., Vorobyeva E. V. Osobennosti vyzvannoi aktivnosti mozga devushek-nositel'nits razlichnykh genotipov po markeru MAOA -uVNTR pri otsenke emotsional'no okrashennykh stsen [Features of the evoked brain activity in female carriers of different MAOA - UVNTR genotypes when assessing emotionally charged scenes]. Rossiiskii psikhologicheskii zhurnal Russian Psychological Journal, 2016, V. 13, no. 4, pp. 232-253.

Original manuscript received 27.10.2016

\section{Введение}

Генетические влияния на особенности функционирования структур нервной системы на сегодняшний день активно изучаются [10, 18, 24]. Развиваются такие направления знаний, как генетика мозга и нейропсихофармакология, призванные ответить на вопрос о роли наследственного материала в формировании особенностей процессов, протекающих в нервной системе. Принимая во внимание постулат о полигенном наследовании сложных функций [9], ученые ведут поиск генов-кандидатов, оказывающих влияние на строение, объем мозговых структур (к примеру - на толщину коры (Chen et al.)), а также - на особенности метаболизма в различных областях мозга и на специфику их функционирования [7, 19]. В последнее время появляются также работы, посвященные изучению влияния специфики генотипа и личностных особенностей на компоненты вызванных потенциалов (ВП) $[1,3,30]$. Однако недостаточно изученным остается механизм, объясняющий связь 


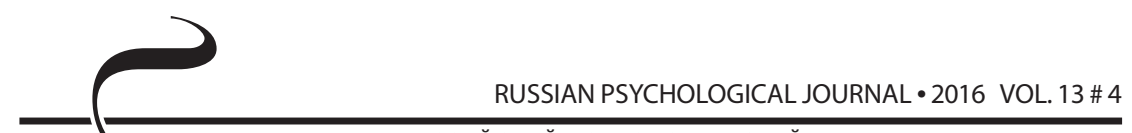

РОССИЙСКИЙ ПСИХОЛОГИЧЕСКИЙ ЖУРНАЛ • 2016 ТОМ 13 № 4

различных генотипов генов нейромедиаторных систем с особенностями вызванной активности мозга в ответ на эмоционально окрашенные стимулы.

Известным является факт воздействия нейротрансмиттеров на интенсивность, продолжительность и полярность эмоциональных реакций $[4,6,10]$. В связи с этим актуальным выступает изучение особенностей мозговой активности у носителей различных генотипов, оказывающих влияние на концентрацию нейромедиаторов в различных областях мозга. К числу генов, ассоциированных с эмоциональными и поведенческими реакциями, а также - с познавательными и личностными особенностями $[5,9,15]$, относится ген моноаминоксидазы А (MAOA), вызывающий интерес у многих ученых за счет влияния на концентрацию дофамина, серотонина, норадреналина и длительность их пребывания в синаптическом пространстве [10, 31, 34].

Ген МАОА имеет структурную вариабельность, проявляющуюся в количестве повторов промотора: носители аллелей с 2, 3, 5 повторами имеют низкоактивную форму гена и фермента, катаболизирующего нейромедиаторы, что приводит к более длительному пребыванию дофамина, норадреналина и серотонина в синаптическом пространстве; обладатели аллелей с 3.5, 4, 3.5/4 повторами имеют высокоактивную моноаминоксидазу А, что приводит к непродолжительному пребыванию моноаминов в синаптическом пространстве. За счет особенностей расположения аллелей гена на X-хромосоме, женщины являются сложным объектом для изучения связи полиморфизмов МАОА с психологическими и психофизиологическими показателями [34], у них возможны гетерозиготные генотипы: 3.5/3, 3/4, 4/5 [5, 31]. Считается, что гетерозиготность делает картину проявления признаков неясной. В последнее время появляются исследования, изучающие эпигенетические воздействия факторов среды на экспрессию генов [11]. Показано, что в зависимости от средовых условий конкурирующие аллели, расположенные на разных Х-хромосомах женщины, могут не только уравновешивать влияние друг друга, как считалось ранее, но и вступать в конкуренцию [34]. Травматический опыт, приобретенный девушками в процессе развития, может существенно влиять на экспрессию генов (в том числе, МАОА), способствуя манифестации непроявленных до определенного времени особенностей поведения (например, импульсивности, антисоциальности) [12]. Таким образом, некоторые исследователи говорят о среднем уровне активности данных генотипов, по сравнению с другими, иные ученые говорят о подавлении одного из аллелей, третьи - о возможности их попеременной активации сообразно условиям окружающей среды.

МАОА оказывает воздействие на деятельность мезолимбической субсистемы дофаминовой системы, которая тесно связана с поясной извилиной, миндалиной, прилежащим ядром, гиппокампом, другими компонентами 
лимбической системы и префронтальной корой $[7,8,9]$. Согласно современным научным представлениям, низко- и высокоактивные генотипы по маркеру MAOA -uVNTR накладывают весомый отпечаток на специфику функционирования перечисленных структур и опосредованно - на эмоциональную сферу человека, на восприятие и оценку им стимулов различной валентности, на механизмы социального поведения, в том числе, - на проявление агрессии $[22,35,36]$. Префронтальные области в данном случае служат в качестве регуляторов импульсов, поступающих от структур лимбической системы, они призваны осуществлять фильтрацию и рациональную оценку различных стимулов. Современные исследования показывают, что связь между миндалиной и префронтальной корой опосредована участием поясной извилины $[14,15,28]$. Таким образом, ген МАОА может принимать участие в формировании особенностей взаимодействия перечисленных структур за счет экспрессии в них.

Целью данного исследования явилось изучение амплитудных и пространственно-временных параметров вызванной активности мозга девушекносительниц различных генотипов по маркеру MAOA -uVNTR в процессе оценки эмоционально окрашенных стимулов.

Гипотеза исследования: носители высокоактивных и гетерозиготных генотипов гена МАОА, вероятно, имеют различия в особенностях амплитудных и пространственно-временных параметров вызванной активности мозга в процессе оценки эмоционально окрашенных стимулов.

\section{Программа исследования}

В состав выборки была включена 61 праворукая девушка в возрасте 18-24 лет. После выделения ДНК из буккального соскоба производилось генотипирование по маркеру MAOA -uVNTR. Таким образом, среди участниц исследования были выделены 2 группы: с высокоактивными $(3.5,4,3.5 / 4)$ генотипами в количестве 38 человек и гетерозиготными $(3.5 / 3,3 / 4,4 / 5)$ генотипами в количестве 23 человек.

Вторым этапом исследования выступила запись ЭЭГ с регистрацией зрительных вызванных потенциалов (ВП). Методом экспертных оценок были выделены группы эмоционально окрашенных стимулов, предъявлявшихся участницам исследования с экрана монитора: «нейтральные», «позитивные», «толерантность», «агрессия», «экстремизм, терроризм». Стимульные изображения были уравнены по физическим параметрам: размеру, цвету, яркости, контрасту. Для записи ЭЭГ использовался электроэнцефалограф NVX-136 («MКС», Россия) с 64 отведениями. Испытуемым была дана инструкция оценивать каждое изображение по его эмоциональной валентности (негативное, нейтральное, позитивное). 
В целях обработки и анализа записей был использован пакет EEGlab для программы MatLab. Для статистической обработки данных был применен t-критерий Стьюдента с поправкой Холма на множественные сравнения; уровень значимости различий $\mathrm{p} \leq 0,001$. Эпоха анализа составила $[-100$; 600 мс]. В качестве контрольной группы стимулов нами были выбраны «нейтральные» картинки; с параметрами ВП в ответ на изображения данного кластера мы сравнивали характеристики вызванной активности мозга носительниц высокоактивных и гетерозиготных генотипов гена МАОА при оценке эмоционально окрашенных стимулов.

\section{Результаты, полученные в группе носительниц высокоактивных генотипов гена МАОА, и их интерпретация}

Сравнительный анализ параметров ВП в ответ на нейтральные стимулы и изображения группы «агрессия». Анализ вызванной активности мозга обладательниц аллелей гена МАОА, несущих 3.5, 4, 3.5/4 повторов исследуемого отрезка ДНК, позволяет сделать вывод о наличии различий в амплитудных характеристиках компонентов ВП в ответ на стимулы группы «агрессия», по сравнению с ВП на нейтральные изображения $(p \leq 0,001)$. При этом вызванные потенциалы сформированы симметрично в структурах правого и левого полушарий (рисунок 1). В районе 150-й мс преобладают процессы возбуждения в нейронных сетях, расположенных в окципитальных отделах, что может быть связано с модальностью предъявляемых стимулов; к 280-й мс фокус активности перемещается в передние и центральные области коры, симметрично распределяясь между правым и левым полушариями, что свидетельствует о равном вкладе обеих гемисфер в процесс когнитивной обработки стимулов. К 380-й мс активно вовлеченными в процесс анализа стимула остаются фронтальные области правой и левой гемисфер. Отсутствие выраженной асимметрии вызванной активности нейронных сетей может быть связано в данном случае с неоднозначной оценкой валентности предъявляемых стимулов, а также - с последовательно-параллельным способом обработки зрительной эмоционально окрашенной информации. Таким образом, у данной группы участниц получены достоверно значимые различия в амплитудных характеристиках средних и поздних компонентов вызванных потенциалов при оценке «нейтральных» и «агрессивных» стимулов.

Сравнительный анализ параметров ВП в ответ на нейтральные стимулы и изображения группы «экстремизм, терроризм». При оценке изображений группы «экстремизм, терроризм», по сравнению с «нейтральными» (рисунок 2), у носительниц высокоактивных генотипов гена МАОА на ранних и средних этапах вызванной активности выражено преобладание окципитальной асимметрии, связанной, вероятно, с концентрацией внимания 
RUSSIAN PSYCHOLOGICAL JOURNAL・2016 VOL. 13 \# 4

на сложных зрительных образах, а также - с процессом их распознавания. В районе 250-350-й мс выявлены достоверно значимые различия $(p \leq 0,001)$ в амплитудных характеристиках ВП с преобладанием активности в височнотеменной области правого полушария, что может быть связано с переживанием участницами негативных эмоций, возникающих в ответ на предъявление стимулов данной группы [16, 25].
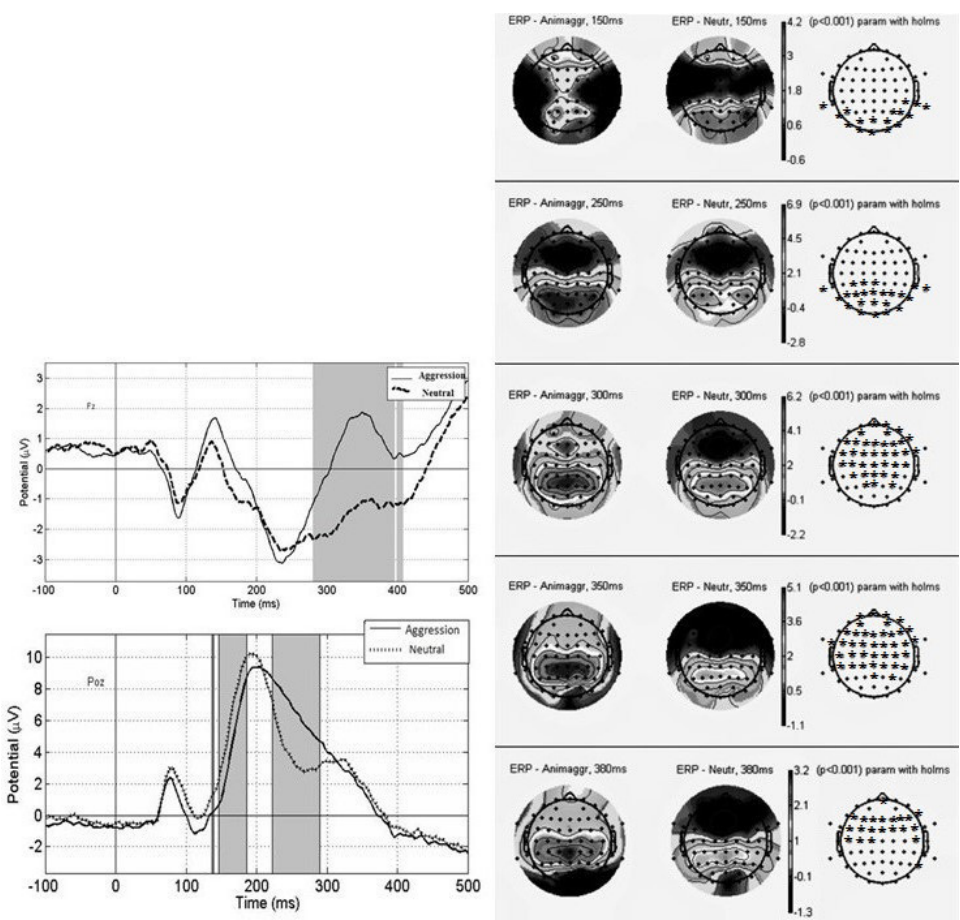

Рисунок 1. ВП в ответ на изображения категорий «агрессия» и «нейтральные» у испытуемых с высокоактивными генотипами гена MAOA

Условные обозначения: серыми полосами (на графиках) и знаком (*) на схеме расположения электродов отмечены достоверно значимые различия в амплитуде/латентности компонентов ВП в ответ на стимулы разных категорий, $p \leq 0,001$.

Figure 1. EP in response to the images of "aggression" and "neutral" categories in the subjects with the high-activity MAOA genotypes

Legend: grey bars (in diagrams) and $\left(^{*}\right)$ signs in the diagram of the electrodes correspond to reliably significant differences in the amplitude/latency of the EP components in response to stimuli of different categories, $p \leq 0.001$. 


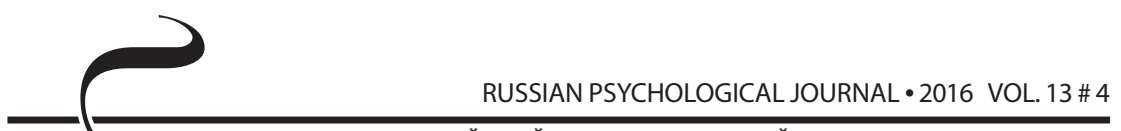

РОССИЙСКИЙ ПСИХОЛОГИЧЕСКИЙ ЖУРНАЛ • 2016 ТОМ 13 № 4
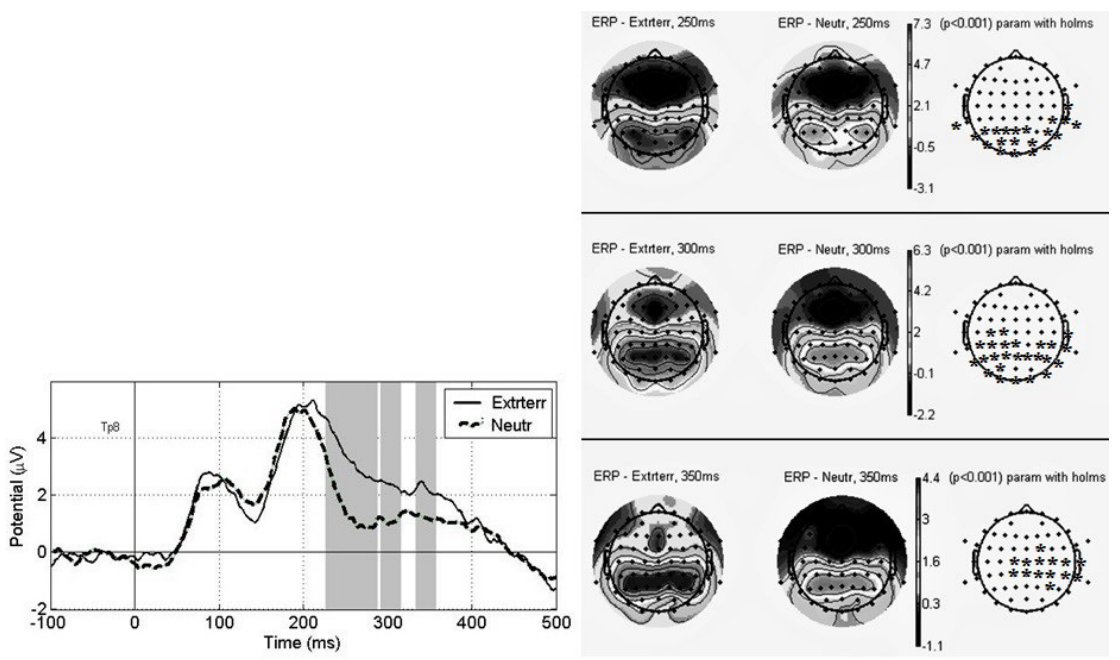

Рисунок 2. ВП в ответ на изображения категорий «экстремизм, терроризм» и «нейтральные» у испытуемых с высокоактивными генотипами гена MAOA

Условные обозначения: серыми полосами (на графиках) и знаком (*) на схеме расположения электродов отмечены достоверно значимые различия в амплитуде/латентности компонентов ВП в ответ на стимулы разных категорий, $p \leq 0,001$.

Figure 2. EP in response to the images of "extremism and terrorism" and "neutral" categories in the subjects with the high-activity MAOA genotypes

Legend: grey bars (in diagrams) and $\left(^{*}\right)$ signs in the diagram of the electrodes correspond to reliably significant differences in the amplitude/latency of the EP components in response to stimuli of different categories, $p \leq 0.001$.

\section{Сравнительный анализ параметров ВП в ответ на нейтральные} стимулы и изображения групn «позитивные», «толерантность». Картина вызванной активности мозга обладательниц высокоактивных генотипов MAOA при оценке изображений групп «позитивные» и «толерантность», в сравнении с «нейтральными», имеет общие черты (рисунок 3). Уже на 120-й мс отмечаются достоверно значимые различия $(p \leq 0,001)$ в амплитуде компонентов ВП: во фронтальных областях со сдвигом влево отмечается рост позитивности, а в темпорально-окципитальных областях со сдвигом вправо отмечается рост негативности при просмотре эмоционально окрашенных изображений, по сравнению с нейтральными. 

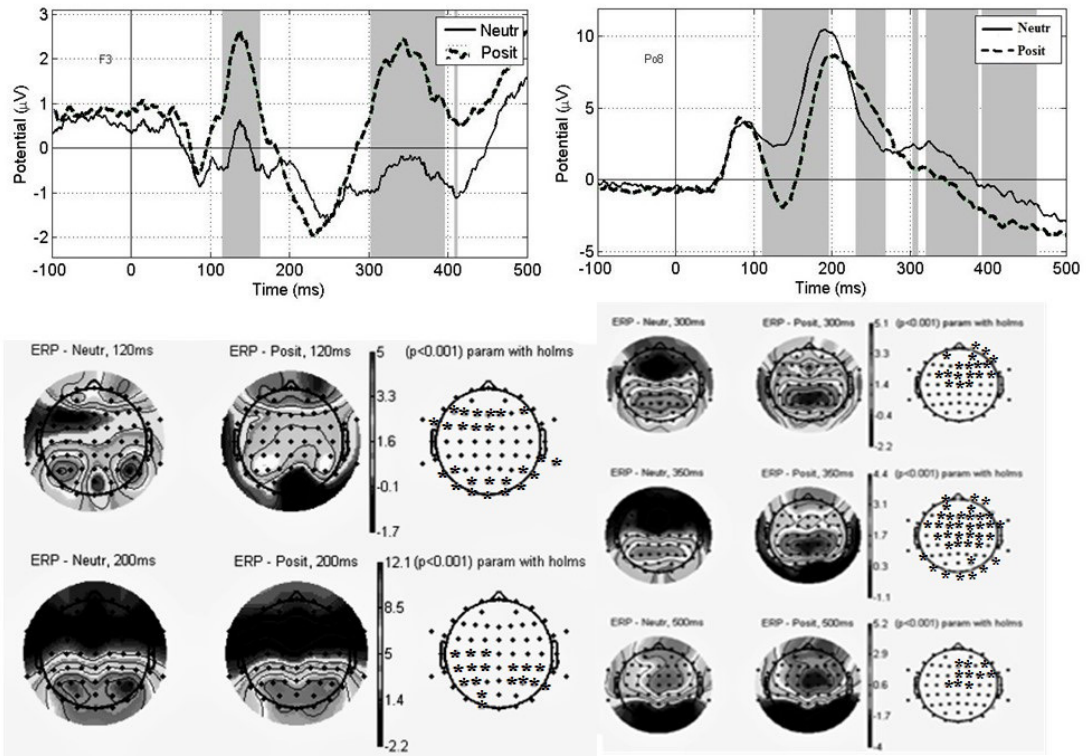

Рисунок 3. ВП в ответ на изображения категорий «позитивные» и «нейтральные» у испытуемых с высокоактивными генотипами гена MAOA

Условные обозначения: серыми полосами (на графиках) и знаком (*) на схеме расположения электродов отмечены достоверно значимые различия в амплитуде/латентности компонентов ВП в ответ на стимулы разных категорий, $p \leq 0,001$.

Figure 3. EP in response to the images of "positive" and "neutral" categories in the subjects with the high-activity MAOA genotypes

Legend: grey bars (in diagrams) and (*) signs in the diagram of the electrodes correspond to reliably significant differences in the amplitude/latency of the EP components in response to stimuli of different categories, $p \leq 0.001$.

Различия в параметрах средних компонентов ВП могут быть связаны с большей активизацией процессов внимания в ответ на предъявление эмоционально окрашенных стимулов (рисунки 3, 4). По мнению D. Talsma с соавт., увеличение амплитуды ранних и средних компонентов ВП отражает интенсификацию процесса восприятия [32]. Активизация фронтальных отделов левой гемисферы на данном этапе обработки стимулов может быть связана с легким распознаванием и ярким переживанием положительных эмоций в ответ на предъявляемые стимулы. Увеличение амплитуды компонентов 


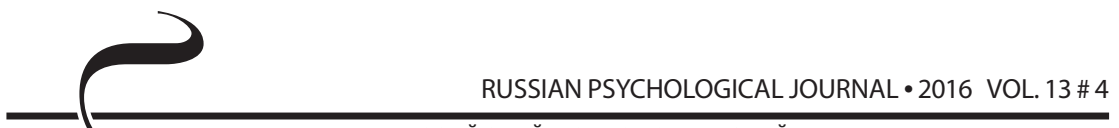

РОССИЙСКИЙ ПСИХОЛОГИЧЕСКИЙ ЖУРНАЛ • 2016 ТОМ 13 № 4

вызванной активности во фронтальных отделах на поздних этапах обработки стимулов может быть связано также с наличием особой, субъективной значимости оцениваемых изображений для испытуемых [33].

Активизация теменно-затылочных областей правого полушария может быть связана с оценкой физических параметров зрительных стимулов (контур, форма, контрастность, сложность и др.), с восприятием и неосознанной оценкой эмоционального контекста изображений, а также с соотнесением их с эпизодами из личного опыта, хранящимися в памяти [26].
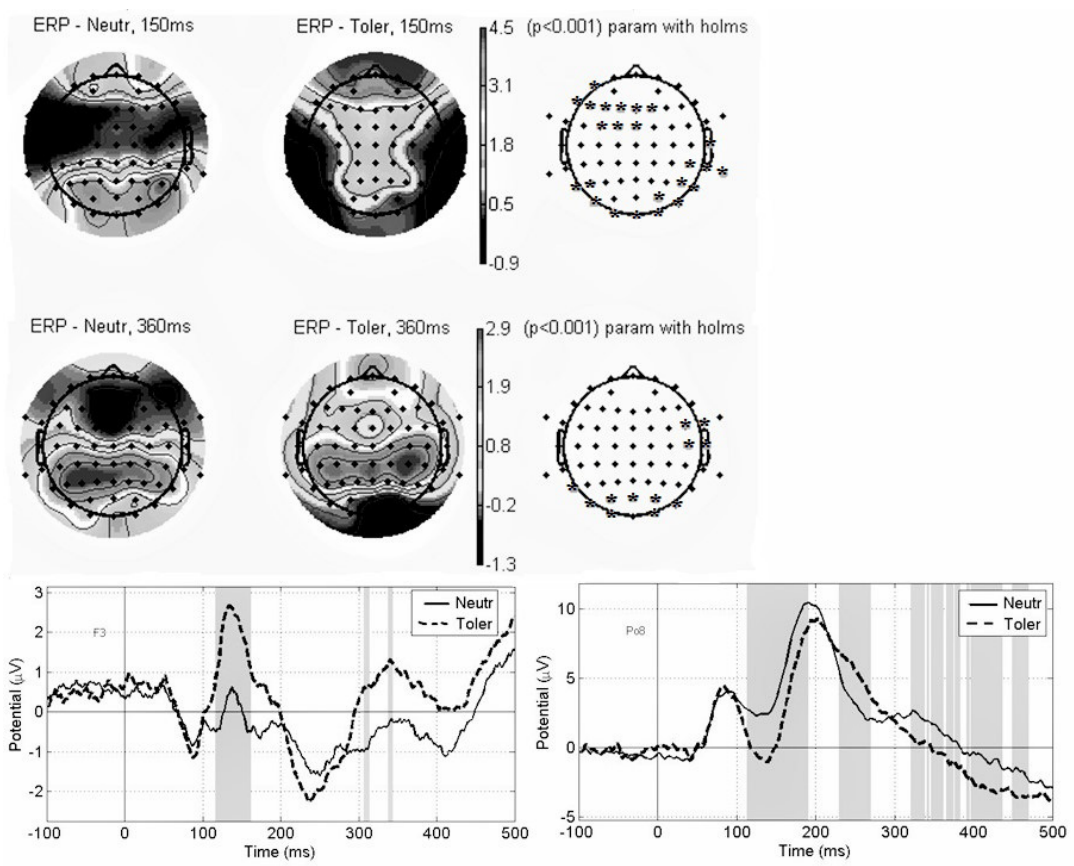

Рисунок 4. ВП в ответ на изображения категорий «толерантность» и «нейтральные» у испытуемых с высокоактивными генотипами гена MAOA

Условные обозначения: серыми полосами (на графиках) и знаком (*) на схеме расположения электродов отмечены достоверно значимые различия в амплитуде/латентности компонентов ВП в ответ на стимулы разных категорий, $p \leq 0,001$.

Figure 4. EP in response to the images of "tolerance" and "neutral" categories in the subjects with the high-activity MAOA genotypes

Legend: grey bars (in diagrams) and $(*)$ signs in the diagram of the electrodes correspond to reliably significant differences in the amplitude/latency of the EP components in response to stimuli of different categories, $p \leq 0.001$. 


\section{Результаты, полученные в группе носительниц гетерозиготных генотипов гена МАОА}

Сравнительный анализ параметров ВП в ответ на нейтральные стимулы и изображения групn "агрессия", "экстремизм, терроризм». Из представленных ниже карт вызванной активности мозга испытуемых с гетерозиготными генотипами гена МАОА видно, что различия в оценке эмоционально окрашенных и нейтральных изображений у них выражены не так интенсивно, как у носительниц высокоактивных форм гена. В ответ на изображения групп «агрессия» и «экстремизм, терроризм», по сравнению с нейтральными, отличия в среднелатентных компонентах вызванной активности локализуются в окципитальных областях, в длиннолатентных компонентах - во фронто-центральных и центрально-париетальных областях ( $\leq \leq 0,001)$ с преимущественной активизацией левого полушария (рисунок 5), что может быть связано с попыткой дать рациональное объяснение возникшим при оценке стимулов эмоциональным реакциям, а также с преобладанием процесса последовательной обработки эмоционально окрашенной информации.

Сравнительный анализ параметров ВП в ответ на нейтральные стимулы и изображения групп «позитивные», «толерантность». При оценке «позитивных» изображений, по сравнению с «нейтральными», отмечаются достоверно значимые различия ( $p \leq 0,001)$ в средних и поздних компонентах ВП во всех областях мозга, за исключением центрально-париетальных отделов. Отмечается тенденция к левосторонней латерализации активности, что может свидетельствовать о положительном знаке эмоций, возникающих при оценке данной группы стимулов, а также - о последовательном способе обработки информации.

Уменьшение амплитуды компонента Р200 зарегистрировано в задних областях мозга носительниц гетерозиготных генотипов гена MAOA при оценке изображений группы «толерантность» (рисунок 6). Данная особенность может свидетельствовать об усилении концентрации внимания по отношению к стимулам данной группы, к специфике элементов изображений $[6,27]$. При этом в лобных и центральных отделах различия, по сравнению с реакцией на нейтральные изображения, найдены не были.

\section{Обсуждение результатов}

Изучая особенности вызванной активности мозга носителей МАОА, мы нашли косвенное подтверждение существующему предположению о наличии связи между спецификой строения гена и управляющими функциями мозга $[17,23,29]$. У девушек с высокоактивными генотипами (непродолжительное пребывание моноаминов в синаптическом пространстве) они более эффективны, по сравнению с носителями гетерозиготных генотипов, что может отражаться на особенностях протекания когнитивных процессов [2]. 

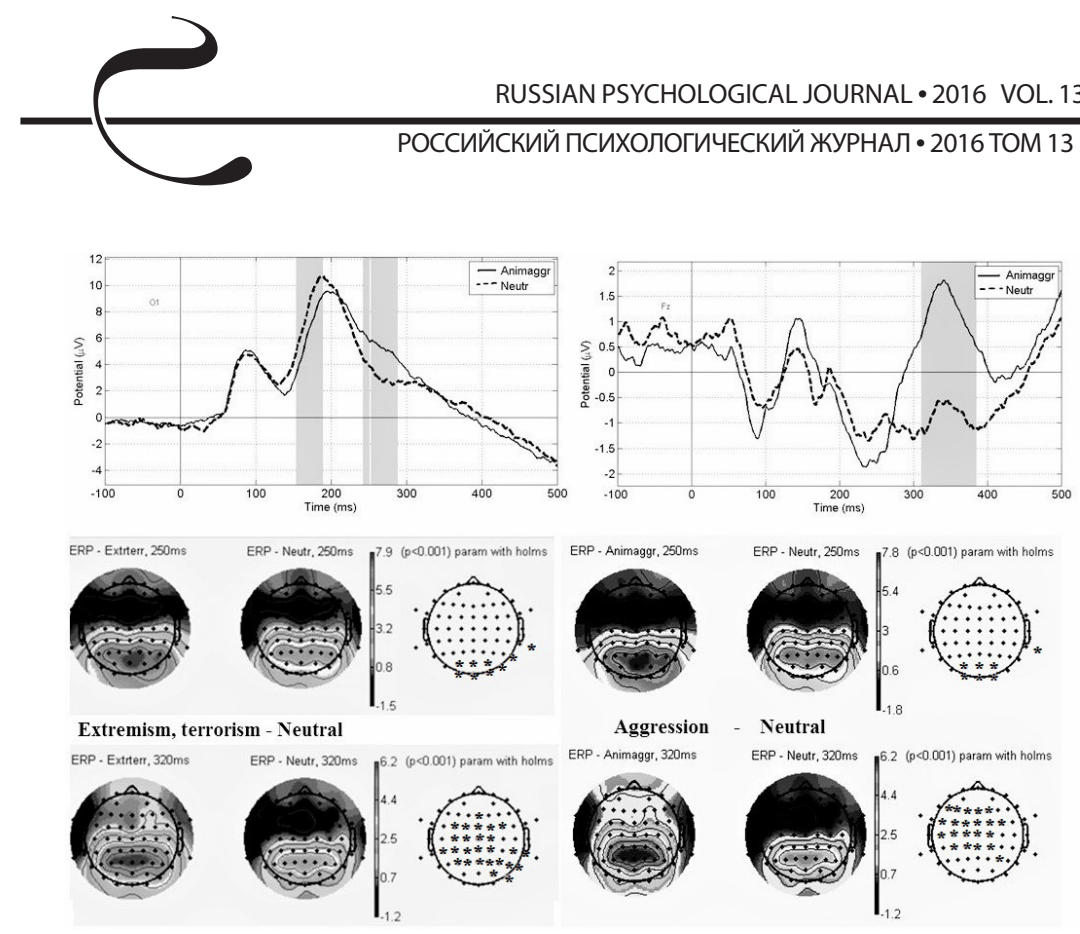

Рисунок 5. ВП в ответ на изображения категорий «агрессия», «экстремизм, терроризм» и «нейтральные» у испытуемых с гетерозиготными генотипами гена МАOA

Условные обозначения: серыми полосами (на графиках) и знаком (*) на схеме расположения электродов отмечены достоверно значимые различия в амплитуде/латентности компонентов ВП в ответ на стимулы разных категорий, $p \leq 0,001$.

Figure 5. EP in response to the images of "aggression", "extremism, terrorism" and "neutral" categories in the subjects with the heterozygous MAOA genotypes

Legend: grey bars (in diagrams) and $\left(^{*}\right)$ signs in the diagram of the electrodes correspond to reliably significant differences in the amplitude/latency of the EP components in response to stimuli of different categories, $p \leq 0.001$.

Представления о влиянии генотипов МАОА на кортико-лимбическое взаимодействие в иерархическом ряду «миндалина - поясная извилина - префронтальная кора» могут быть объяснены следующим образом: повышение содержания моноаминов (в т. ч. дофамина) в префронтальных отделах способствует чрезмерной их активации, развитию тревоги и снижению управляющих функций (что свойственно лицам с низкоактивными генотипами, не вошедшим в состав нашей выборки) [8, 13, 21, 32]. Лица с высокоактивными генотипами, наоборот, имеют низкое содержание моноаминов в префронтальной коре, что способствует лучшему выполнению задач с вовлечением функций внимания, т. к. возбуждение происходит в моменты, 
когда необходимо принять решение, сделать выбор [20]. Таким образом, поступающие стимулы и задачи подвергаются более тщательной фильтрации.

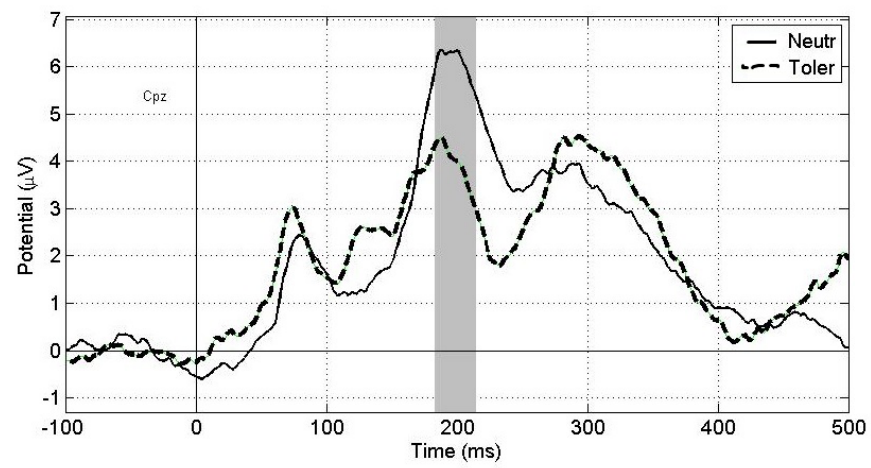

Рисунок 6. ВП в ответ на изображения категорий «толерантность» и «нейтральные» у испытуемых с гетерозиготными генотипами MAOA Условные обозначения: серыми полосами отмечены достоверно значимые различия в амплитуде компонентов ВП в ответ на стимулы разных категорий, $p \leq 0,001$.

Figure 6. EP in response to the images of "tolerance", "neutral" categories in the subjects with the heterozygous MAOA genotypes

Legend: grey bars correspond to reliably significant differences in the amplitude the EP components in response to stimuli of different categories, $p \leq 0.001$.

Интересным является анализ особенностей вызванной активности мозга у носителей гетерозиготных генотипов МАОА. Принимая во внимание полученные результаты, мы можем заключить, что активность префронтальных отделов мозга данной группы участниц имеет особенности, заключающиеся в выборочной активации перечисленных мозговых структур (она происходит в случае, если внешний стимул производит сильный возбуждающий эффект). В обработке других стимулов в большей степени участвуют задние отделы мозга. При этом активация центрально-париетальных отделов, отвечающих за межмодальный синтез, согласно полученным нами результатам, у данной группы протекает избирательно, в отличие от носителей высокоактивных генотипов.

\section{Выводы}

На основании вышеизложенного мы можем заключить, что:

1. Строение гена MAOA, за счет влияния на длительность пребывания нейромедиаторов-моноаминов в синаптическом пространстве и активность процессов, протекающих в нейромедиаторных системах (в т. ч. 


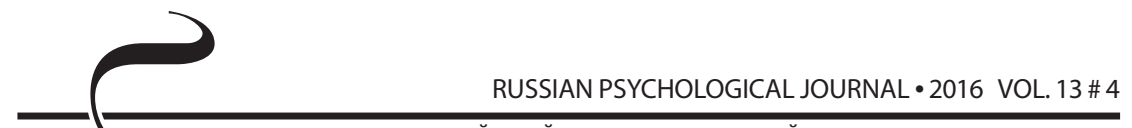

РОССИЙСКИЙ ПСИХОЛОГИЧЕСКИЙ ЖУРНАЛ • 2016 ТОМ 13 № 4

в мезолимбической подсистеме дофаминовой системы), с высокой вероятностью ассоциировано с особенностями вызванной активности мозга при оценке эмоционально окрашенных сцен.

2. Параметры вызванной активности мозга при просмотре нейтральных и эмоционально окрашенных изображений у девушек с высокоактивными генотипами МАОА имеют больше отличий, по сравнению с носителями гетерозиготных вариантов.

3. Активизация процессов когнитивной обработки эмоционально значимых стимулов у обладательниц высокоактивных генотипов МАОА протекает более интенсивно, что проявляется в различиях параметров поздних компонентов вызванной активности коры обеих гемисфер при оценке всех групп эмоционально окрашенных стимулов, по сравнению с нейтральными.

Увеличение амплитуды компонентов N1 во фронто-центральных областях левого полушария и Р1 - в париетально-окципитальных областях правого полушария при просмотре изображений групп «позитивные» и «толерантность» может свидетельствовать об усилении функционирования таких параметров внимания, как селективность и концентрация [32, 33].

4. Межмодальный синтез у обладательниц гетерозиготных генотипов MAОА, по-видимому, протекает менее эффективно, нежели у обладательниц высокоактивных форм гена. Это проявляется в том, что, по сравнению с носительницами высокоактивных генотипов МАОА, у обладательниц гетерозиготных генотипов при просмотре нейтральных и эмоционально окрашенных стимулов не имеется достоверно значимых различий в параметрах активации нейронных систем центрально-париетальных областей, ответственных за синтез информации разных модальностей $[6,27]$.

5. Для активации нейронных систем лобных отделов девушкам, имеющим гетерозиготные генотипы МАОА, необходимо воздействие раздражителей, обладающих высоким arousal-эффектом. Вероятно, отбор релевантных стимулов у представительниц данной группы происходит менее эффективно [20, 32].

Данный вывод подтверждается отсутствием значимых различий в параметрах вызванной активности фронтальных и центральных отделов мозга при оценке изображений, имеющих более сложный смысловой контекст («экстремизм, терроризм» и «толерантность»): достоверно значимые различия в амплитудно-временных параметрах вызванной активности мозга, по сравнению с параметрами ВП в ответ на нейтральные изображения, у представительниц описываемой группы проявляются только в задних отделах мозга (что может быть объяснено активацией процессов зрительной обработки), в то время как при оценке стимулов, имеющих более понятный контекст и обладающих большим возбуждающим эффектом, различия наблюдаются во фронто-центральных и париетально-окципитальных областях обоих полушарий. 
Таким образом, мы можем заключить, что девушки, в синаптическом пространстве которых нейромедиаторы-моноамины пребывают непродолжительный промежуток времени, демонстрируют большую эмоциональную реактивность, более эффективные показатели избирательности и концентрации внимания $[6,27]$. Успешное функционирование внимания, в свою очередь, способствует более точному отбору релевантной информации [23], а также - ее адекватной оценке и построению программ поведения (что находит отражение в сложном рисунке вызванных потенциалов в ответ на эмоционально окрашенные стимулы, в противовес нейтральным, во всех областях коры мозга).

В заключение необходимо отметить, что генетические влияния имеют сложный и до конца не изученный механизм воздействия на различные системы организма [11]. Данное исследование позволило впервые описать психофизиологические фенотипы обладательниц высокоактивных и гетерозиготных генотипов МАОА. Тем не менее, делать точные выводы о наличии взаимосвязей между генотипами и психофизиологическими признаками можно лишь при учете широкого числа факторов: пола, гормонального фона человека, влияний других генов, особенностей индивидуальной и общей среды, наличия травматического опыта и др. [34]. Перспективой данного исследования выступает комплексное изучение указанных факторов в их взаимосвязи.

\section{Литература}

1. Алфимова М. В., Голимбет В. Е. Гены и нейрофизиологические показатели когнитивных процессов: обзор исследований // Журнал высшей нервной деятельности им. И. П. Павлова. - 2011. - Т. 61. - № 4. - С. 389-401.

2. Алфимова М. В., Голимбет В. Е., Егорова М. С. Личностные черты, управляющие функции и генетические особенности метаболизма моноаминов // Психология. Журнал Высшей школы экономики. - 2009. - Т. 6. - № 4.

3. Алфимова М. В., Голимбет В. Е., Лебедева И. С., Коровайцева Г. И., Лежейко Т. В. Влияние тревожности и гена СОМТ на вызванные потенциалы мозга и продуктивность избирательного внимания // Журнал высшей нервной деятельности им. И. П. Павлова. - 2014. - Т. 64. - № 3. - С. 270. Doi:10.7868/s0044467714030034

4. Богданова И. В. Роль дофамина в механизмах формирования некоторых расстройств ЦНС и состояний зависимости (обзор литературы) //Український вісник психоневрології. - 2011. - № 19. - вип. 2. - С. 5-8.

5. Егорова М. С., Черткова Ю. Д. Полиморфизм гена моноаминооксидазы (МАОА) и вариативность психологических черт // Психологические исследования: электрон. науч. журн. - 2011. - № 6 (20). - C. 14. - URL: http://psystudy.ru 
6. Коваленко А. А., Павленко В. Б. Эмоциональная значимость стимула и черты личности: отражение в паттерне вызванных ЭЭГ-потенциалов // Нейрофизиология. - 2009. - С. 336-356.

7. Шабанов П. Д., Лебедев А. А. Нейрохимические механизмы прилежащего ядра, реализующие подкрепляющие эффекты самостимуляции латерального гипоталамуса // Медицинский академический журнал. 2012. - T. 12. - № 2. - С. 68-76.

8. Barnes J. M., Dean A. J., Nandam L. S., O'Connell R. G., Bellgrove M. A. The Molecular Genetics of Executive Function: Role of Monoamine System Genes // Biological Psychiatry. - 2011. - V. 69. - I. 12. - pp. 127-143. Doi:10.1016/j.biopsych.2010.12.040

9. Barnett J. H., Xu K., Heron J., Goldman D., Jones P. B. Cognitive effects of genetic variation in monoamine neurotransmitter systems: a populationbased study of COMT, MAOA and 5HTTLPR // American Journal of Medical Genetics. Part B: Neuropsychiatric Genetics. - 2011. - V. 156. - pp. 158-167. Doi:10.1002/ajmg.b.31150

10. Basic Neurochemistry: Principles of Molecular, Cellular and Medical Neurobiology / Ed. by Brady S. T. et al. - Academic press / Elsevier, 2012. - 1096 p. Doi:10.1212/wnl.39.3.460-b

11. Battaglia M. Challenges in the appraisal and application of gene-environment interdependence // European Journal of Developmental Psychology. 2012. - V. 9. - no. 4. - pp. 419-425. Doi:10.1080/17405629.2012.689819

12. Beach S. R., Brod G. H., Gunter T. D., Packer H., Wernett P., Philibert R. A. Child maltreatment moderates the association of MAOA with symptoms of depression and antisocial personality disorder // Journal of Family Psychology. - 2010. - V. 24 (1). - pp. 12-20. doi:10.1037/a0018074

13. Bishop S. J. Trait anxiety and impoverished prefrontal control of attention // Nature neuroscience. - 2009. - V. 12. - no. 1. - pp. 92-98. Doi:10.1038/ $\mathrm{nn} .2242$

14. Buckholtz J., Callicott J., Kolachana B., Hariri A., Goldberg T., Genderson M. Genetic variation in MAOA modulates ventromedial prefrontal circuitry mediating individual differences in human personality // Molecular Psychiatry. - 2008. - V. 13. - pp. 313-324. Doi:10.1038/sj.mp.4002020

15. Buckholtz J. W., Meyer-Lindenberg A. MAOA and the neurogenetic architecture of human aggression // Trends in neurosciences. - 2008. - V. 31.3. pp. 120-129. Doi:10.1016/j.tins.2007.12.006

16. Cacioppo J. T., Crites S. L., Gardner W. L. Attitudes to the right: Evaluative processing is associated with lateralized late positive event-related brain potentials // Personality and Social Psychology Bulletin. - 1996. - V. 22. no. 12. - pp. 1205-1219. Doi:10.1177/01461672962212002 
17. Cerasa A., Gioia M. C., Fera F., Passammonti L., Liguori M., Lanza P., Muglia M., Maagariello A., Quattrone A. Ventrolateral prefrontal activity during working memory is modulated by MAO A genetic variation // Brain Res. 2008. - V. 1201. - pp. 114-121. Doi:10.1016/j.brainres.2008.01.048

18. Chen C. H., Fiecas M., Gutierrez E. D., Panizzon M. S., Eyler L. T., Vuoksimaa E., Neale M. C. Genetic topography of brain morphology // Proceedings of the National Academy of Sciences. - 2013. - V. 110. - no. 42. - pp. 17089-17094. Doi:10.1073/pnas.1308091110

19. Chen J., Lipska B. K., Halim N., Ma Q. D., Matsumoto M., Melhem S. Functional analysis of genetic variation in catechol-O-methyltransferase (COMT): Effects on $\mathrm{mRNA}$, protein, and enzyme activity in postmortem human brain //The American Journal of Human Genetics. - 2004. - V. 75 (5). - pp. 807-821. doi:10.1086/425589

20. Delplanque S., Lavoie M. E., Hot P., Silvert L., Sequeira H. Modulation of cognitive processing by emotional valence studied through event-related potentials in humans // Neuroscience letters. - 2004. - V. 356. - no. 1. - pp. 1-4. Doi:10.1016/j.neulet.2003.10.014

21. Domschke K., Dannlowski U. Imaging genetics of anxiety disorders // Neuroimage. - 2010. - V. 53. - no. 3. - pp. 822-831. Doi:10.1093/ med/9780199920211.003.0014

22. Ficks C. A., Waldman I. D. Candidate genes for aggression and antisocial behavior: a meta-analysis of association studies of the $5 \mathrm{HTTLPR}$ and MAOA -uVNTR// Behav Genet. - 2014. - V. 44 (5). - pp. 427-444. Doi:10.1007/s10519-014-9661-y

23. Fossella J., Sommer T., Fan J., Wu Y., Swanson J. M., Pfaff D. W., Posner M. I. Assessing the molecular genetics of attention networks // BMC Neuroscience. - 2002. - V.3. - pp. 14-19.Doi:10.1002/9780470015902.a0006012.pub2

24. Goldman D., Oroszi G., Ducci F. The genetics of addictions: Uncovering the genes // Nature Reviews Genetics. - 2005. - V. 6 (7). - pp. 521-532. doi:10.1038/nrg1635

25. Kayser J., Bruder G. E., Tenke C. E., Stewart J. E., Quitkin F. M. Event-related potentials (ERPs) to hemifield presentations of emotional stimuli: differences between depressed patients and healthy adults in P3 amplitude and asymmetry // International Journal of Psychophysiology. - 2000. - V. 36. no. 3. - pp. 211-236. Doi:10.1016/0304-3940(93)90241-c

26. Morgan M. A., Romanski L. M., LeDoux J. E. Extinction of emotional learning: contribution of medial prefrontal cortex // Neuroscience letters. 1993. - V. 163. - no. 1. - pp. 109-113. Doi:10.1016/0304-3940(93)90241-c

27. Olofsson J. K., Nordin S., Sequeira H., Polich J. Affective picture processing: an integrative review of ERP findings // Biological psychology. - 2008. - V. 77. no. 3. - pp. 247-265. Doi:10.1016/j.biopsycho.2007.11.006 
28. Pardini M., Krueger F., Hodgkinson C., Raymont V., Ferrier C., Goldman D., Grafman J. Prefrontal cortex lesions and MAO-A modulate aggression in penetrating traumatic brain injury // Neurology. - 2011. - V. 76 (12). - pp. 1038-1045. http://doi.org/10.1212/WNL.0b013e318211c33e

29. Passamonti L., Fera F., Magariello A., Ceerasa A., Gioia M. C., Muglia M., Nicoletti G., Gallo O., Provinciali L., Quattrone A. Monoamine oxidase-A genetic variations influence brain activity associated with inhibitory control: new insight into the neural correlates of impulsivity // Biol. Psychiatry. - 2006. V. 59. - pp. 334-340. Doi:10.1016/j.biopsych.2005.07.027

30. Smolka M. N., Schumann G., Wrase J., Grüsser S. M., Flor H., Mann K., Heinz A. Catechol-O-methyltransferase val158met genotype affects processing of emotional stimuli in the amygdala and prefrontal cortex // The Journal of Neuroscience. - 2005. - V. 25. - no. 4. - pp. 836-842. Doi:10.1523/jneurosci.1792-04.2005

31. Takahashi A., Quadros I. M., de Almeida R. M., Miczek K. A. Behavioral and pharmacogenetics of aggressive behavior // Behavioral Neurogenetics. Current Topics in Behavioral Neurosciences. - 2012. - V. 12. - pp. 73-138. Doi:10.1007/7854_2011_191

32. Talsma D., Mulckhuyse M., Slagter H. A., Theeuwes J. Faster, more intense! The relation between electrophysiological reflections of attentional orienting, sensory gain control, and speed of responding // Brain Research. - 2007. V. 1178. - pp. 92-105. Doi:10.1016/j.brainres.2007.07.099

33. Taylor M. J. Non-spatial attentional effects on P1 // Clin. Neurophysiol. 2002. - V. 113. - no. 12. - pp. 1903-1908. Doi:10.1016/s1388-2457(02)00309-7

34. Weeland J., Overbeek G., de Castro B. O., \& Matthys W. Underlying Mechanisms of Gene-Environment Interactions in Externalizing Behavior: A Systematic Review and Search for Theoretical Mechanisms // Clinical child and family psychology review. - 2015. - V. 18. - no. 4. - pp. 413-442. http://doi. org/10.1007/s10567-015-0196-4

35. Williams L. M., Gatt J. M., Kuan S. A., Dobson-Stone C., Palmer D. M., Paul R. H., Le Song, Costa P. T., Schofield P. R., Gordon E. A Polymorphism of the MAOA Gene is Associated with Emotional Brain Markers and Personality Traits on an Antisocial Index // Neuropsychopharmacology. - 2009. - no. 34. pp. 1797-1809. Doi:10.1038/npp.2009.1

36. Ziermans T., Dumontheil l., Roggeman C., Peyrard-Janvid M., Matsson H., Kere J., Klingberg T. Working memory brain activity and capacity link MAOA polymorphism to aggressive behavior during development // Citation: Transl. Psychiatry. - 2012. - V. 2. - P. 1038. Doi:10.1038/tp.2012.7 


\section{References}

1. Alfimova M. V., Golimbet V. E. Geny i neirofiziologicheskie pokazateli kognitivnykh protsessov: obzor issledovanii [Genes and neurophysiological characteristics of cognitive processes: a review of research]. Zhurnal vysshei nervnoi deyatel'nosti im. I. P. Pavlova - I. P. Pavlov Journal of Higher Nervous Activity, 2011, V. 61, no. 4, pp. 389-401.

2. Alfimova M. V., Golimbet V. E., Egorova M. S. Lichnostnye cherty, upravlyayushchie funktsii i geneticheskie osobennosti metabolizma monoaminov [Personality traits, control functions, and genetic characteristics of monoamine metabolism]. Psikhologiya. Zhurnal Vysshei shkoly ekonomiki - Psychology. Journal of the Higher School of Economics, 2009, V. 6, no. 4.

3. Alfimova M. V., Golimbet V. E., Lebedeva I. S., Korovaitseva G. I., Lezheiko T. V. Vliyanie trevozhnosti i gena COMT na vyzvannye potentsialy mozga i produktivnost' izbiratel'nogo vnimaniya [The influence of anxiety and the COMT gene on evoked potentials and the productivity of selective attention]. Zhurnal vysshei nervnoi deyatel'nosti im. I. P. Pavlova - I. P. Pavlov Journal of Higher Nervous Activity, 2014, V. 64, no. 3, P. 270. Doi:10.7868/ s0044467714030034

4. Bogdanova I. V. Rol' dofamina v mekhanizmakh formirovaniya nekotorykh rasstroistv TsNS i sostoyanii zavisimosti (obzor literatury) [The role of dopamine in the mechanisms of formation of certain disorders of the central nervous system and states of addiction (a literature survey)]. Ukraïn'kii visnik psikhonevrologii - Ukrainian Bulletin of Psychoneurology, 2011, no. 19, V. 2, pp. 5-8.

5. Egorova M. S., Chertkova Yu. D. The monoamine oxidase (MAOA) gene polymorphism and the variability of psychological traits. Psychological research, 2011, no. 6 (20), P. 14. Available at: http://psystudy.ru

6. Kovalenko A. A., Pavlenko V. B. Emotsional'naya znachimost' stimula i cherty lichnosti: otrazhenie v patterne vyzvannykh EEG-potentsialov [Emotional significance of the stimulus and personality traits: the reflections in the pattern of evoked EEG potentials]. Neirofiziologiya - Neurophysiology, 2009, pp. 336-356.

7. Shabanov P. D., Lebedev A. A. Neirokhimicheskie mekhanizmy prilezhashchego yadra, realizuyushchie podkreplyayushchie effekty samostimulyatsii lateral'nogo gipotalamusa [The neurochemical mechanisms of the nucleus accumbens carrying out reinforcing effects of lateral hypothalamic self-stimulation]. Meditsinskii akademicheskii zhurnal - Medical Academic Journal, 2012, V. 12, no. 2, pp. 68-76.

8. Barnes J. M., Dean A. J., Nandam L. S., O'Connell R. G., Bellgrove M. A. The Molecular Genetics of Executive Function: Role of Monoamine System Genes. Biological Psychiatry, 2011, V.69, I. 12, pp. 127-143. Doi:10.1016/j.biopsych.2010.12.040 
9. Barnett J. H., Xu K., Heron J., Goldman D., Jones P. B. Cognitive effects of genetic variation in monoamine neurotransmitter systems: a population-based study of COMT, MAOA and 5HTTLPR. American Journal of Medical Genetics. Part B: Neuropsychiatric Genetics, 2011,V. 156, pp. 158-167. Doi:10.1002/ajmg.b.31150

10. Basic Neurochemistry: Principles of Molecular, Cellular and Medical Neurobiology. Ed. by Brady S. T. et al. Academic press / Elsevier, 2012. 1096 p. Doi:10.1212/wnl.39.3.460-b

11. Battaglia M. Challenges in the appraisal and application of gene-environment interdependence. European Journal of Developmental Psychology, 2012, V. 9, no. 4, pp. 419-425. Doi:10.1080/17405629.2012.689819

12. Beach S. R., Brod G. H., Gunter T. D., Packer H., Wernett P., Philibert R. A. Child maltreatment moderates the association of MAOA with symptoms of depression and antisocial personality disorder. Journal of Family Psychology, 2010, V. 24 (1), pp. 12-20. doi:10.1037/a0018074

13. Bishop S. J. Trait anxiety and impoverished prefrontal control of attention. Nature neuroscience, 2009, V. 12, no. 1, pp. 92-98. Doi:10.1038/nn.2242

14. Buckholtz J., Callicott J., Kolachana B., Hariri A., Goldberg T., Genderson M. Genetic variation in MAOA modulates ventromedial prefrontal circuitry mediating individual differences in human personality. Molecular Psychiatry, 2008, V. 13, pp. 313-324. Doi:10.1038/sj.mp.4002020

15. Buckholtz J. W., Meyer-Lindenberg A. MAOA and the neurogenetic architecture of human aggression. Trends in neurosciences, 2008, V. 31.3, pp. 120-129. Doi:10.1016/j.tins.2007.12.006

16. Cacioppo J.T., Crites S. L., Gardner W. L. Attitudes to the right: Evaluative processing is associated with lateralized late positive event-related brain potentials. Personality and Social Psychology Bulletin, 1996, V. 22, no. 12, pp. 1205-1219. Doi:10.1177/01461672962212002

17. Cerasa A., Gioia M. C., Fera F., Passammonti L., Liguori M., Lanza P., Muglia M., Maagariello A., Quattrone A. Ventrolateral prefrontal activity during working memory is modulated by MAO A genetic variation. Brain Res., 2008, V. 1201, pp. 114-121. Doi:10.1016/j.brainres.2008.01.048

18. Chen C. H., Fiecas M., Gutierrez E. D., Panizzon M. S., Eyler L. T., Vuoksimaa E., Neale M. C. Genetic topography of brain morphology. Proceedings of the National Academy of Sciences, 2013, V. 110, no. 42, pp. 17089-17094. Doi:10.1073/pnas.1308091110

19. Chen J., Lipska B. K., Halim N., Ma Q. D., Matsumoto M., Melhem S. Functional analysis of genetic variation in catechol-O-methyltransferase (COMT): Effects on mRNA, protein, and enzyme activity in postmortem human brain. The American Journal of Human Genetics, 2004, V. 75 (5), pp. 807-821. doi:10.1086/425589 
20. Delplanque S., Lavoie M. E., Hot P., Silvert L., Sequeira H. Modulation of cognitive processing by emotional valence studied through event-related potentials in humans. Neuroscience letters, 2004, V. 356, no. 1, pp. 1-4. Doi:10.1016/j.neulet.2003.10.014

21. Domschke K., Dannlowski U. Imaging genetics of anxiety disorders. Neuroimage, 2010,V.53, no.3, pp.822-831.Doi:10.1093/med/9780199920211.003.0014

22. Ficks C. A., Waldman I. D. Candidate genes for aggression and antisocial behavior: a meta-analysis of association studies of the 5HTTLPR and MAOA - UVNTR. Behav Genet., 2014, V. 44 (5), pp. 427-444. Doi:10.1007/s10519-014-9661-y

23. Fossella J., Sommer T., Fan J., Wu Y., Swanson J. M., Pfaff D. W., Posner M. I. Assessing the molecular genetics of attention networks. BMCNeuroscience, 2002, V.3, pp. 14-19. Doi:10.1002/9780470015902.a0006012.pub2

24. Goldman D., Oroszi G., Ducci F. The genetics of addictions: Uncovering the genes. Nature Reviews Genetics, 2005, V. 6 (7), pp. 521-532. doi:10.1038/nrg1635

25. Kayser J., Bruder G. E., Tenke C. E., Stewart J. E., Quitkin F. M. Event-related potentials (ERPs) to hemifield presentations of emotional stimuli: differences between depressed patients and healthy adults in P3 amplitude and asymmetry. International Journal of Psychophysiology, 2000, V. 36, no. 3, pp. 211-236. Doi:10.1016/0304-3940(93)90241-c

26. Morgan M. A., Romanski L. M., LeDoux J. E. Extinction of emotional learning: contribution of medial prefrontal cortex. Neuroscience letters, 1993, V. 163, no. 1, pp. 109-113. Doi:10.1016/0304-3940(93)90241-c

27. Olofsson J. K., Nordin S., Sequeira H., Polich J. Affective picture processing: an integrative review of ERP findings. Biological psychology, 2008, V. 77, no. 3, pp. 247-265. Doi:10.1016/j.biopsycho.2007.11.006

28. Pardini M., Krueger F., Hodgkinson C., Raymont V., Ferrier C., Goldman D., Grafman J. Prefrontal cortex lesions and MAO-A modulate aggression in penetrating traumatic brain injury. Neurology, 2011, V. 76 (12), pp. 1038-1045. http://doi.org/10.1212/WNL.0b013e318211c33e

29. Passamonti L., Fera F., Magariello A., Ceerasa A., Gioia M. C., Muglia M., Nicoletti G., Gallo O., Provinciali L., Quattrone A. Monoamine oxidase-A genetic variations influence brain activity associated with inhibitory control: new insight into the neural correlates of impulsivity. Biol. Psychiatry, 2006, V. 59, pp. 334-340. Doi:10.1016/j.biopsych.2005.07.027

30. Smolka M. N., Schumann G., Wrase J., Grüsser S. M., Flor H., Mann K., Heinz A. Catechol-O-methyltransferase val158met genotype affects processing of emotional stimuli in the amygdala and prefrontal cortex. The Journal of Neuroscience, 2005, V. 25, no. 4, pp. 836-842. Doi:10.1523/jneurosci.1792-04.2005

31. Takahashi A., Quadros I. M., de Almeida R. M., Miczek K. A. Behavioral and pharmacogenetics of aggressive behavior. Behavioral Neuroge- 
netics. Current Topics in Behavioral Neurosciences, 2012, V. 12, pp. 73-138. Doi:10.1007/7854_2011_191

32. Talsma D., Mulckhuyse M., Slagter H. A., Theeuwes J. Faster, more intense! The relation between electrophysiological reflections of attentional orienting, sensory gain control, and speed of responding. Brain Research, 2007, V. 1178, pp. 92-105. Doi:10.1016/j.brainres.2007.07.099

33. Taylor M. J. Non-spatial attentional effects on P1. Clin. Neurophysiol., 2002, V. 113, no. 12, pp. 1903-1908. Doi:10.1016/s1388-2457(02)00309-7

34. Weeland J., Overbeek G., de Castro B. O., \& Matthys W. Underlying Mechanisms of Gene-Environment Interactions in Externalizing Behavior: A Systematic Review and Search for Theoretical Mechanisms. Clinical child and family psychology review, 2015, V. 18, no. 4, pp. 413-442. http://doi. org/10.1007/s10567-015-0196-4

35. Williams L. M., Gatt J. M., Kuan S. A., Dobson-Stone C., Palmer D. M., Paul R. H., Le Song, Costa P. T., Schofield P. R., Gordon E. A Polymorphism of the MAOA Gene is Associated with Emotional Brain Markers and Personality Traits on an Antisocial Index. Neuropsychopharmacology, 2009, no. 34, pp. 1797-1809. Doi:10.1038/npp.2009.1

36. Ziermans T., Dumontheil I., Roggeman C., Peyrard-Janvid M., Matsson H., Kere J., Klingberg T. Working memory brain activity and capacity link MAOA polymorphism to aggressive behavior during development. Citation: Transl. Psychiatry, 2012, V. 2, P. 1038. Doi:10.1038/tp.2012.7 